\title{
Electronic correlations at paramagnetic (001) and (110) NiO surfaces: Charge-transfer and Mott-Hubbard-type gaps at the surface and subsurface of (110) NiO
}

\author{
I. Leonov $\oplus^{1,2,3, *}$ and S. Biermann ${ }^{4,5,6, \dagger}$ \\ ${ }^{1}$ M. N. Miheev Institute of Metal Physics, Russian Academy of Sciences, 620108 Yekaterinburg, Russia \\ ${ }^{2}$ Institute of Physics and Technology, Ural Federal University, 620002 Yekaterinburg, Russia \\ ${ }^{3}$ Skolkovo Institute of Science and Technology, 143026 Moscow, Russia \\ ${ }^{4}$ CPHT, CNRS, Ecole Polytechnique, IP Paris, F-91128 Palaiseau, France \\ ${ }^{5}$ Collège de France, 11 place Marcelin Berthelot, 75005 Paris, France \\ ${ }^{6}$ Department of Physics, Division of Mathematical Physics, Lund University, Professorsgatan 1, 22363 Lund, Sweden
}

(Received 23 November 2020; revised 8 March 2021; accepted 11 March 2021; published 7 April 2021)

\begin{abstract}
We explore the interplay of electron-electron correlations and surface effects in the prototypical correlated insulating material, $\mathrm{NiO}$. In particular, we compute the electronic structure, magnetic properties, and surface energies of the (001) and (110) surfaces of paramagnetic NiO using a fully charge self-consistent DFT+ dynamical mean-field theory method. Our results reveal a complex interplay between electronic correlations and surface effects in $\mathrm{NiO}$, with the electronic structure of the (001) and (110) $\mathrm{NiO}$ surfaces being significantly different from that in bulk $\mathrm{NiO}$. We obtain a sizable reduction of the band gap at the surface of $\mathrm{NiO}$, which is most significant for the (110) $\mathrm{NiO}$ surface. This suggests a higher catalytic activity of the (110) $\mathrm{NiO}$ surface than that of the (001) $\mathrm{NiO}$ one. Our results reveal a charge-transfer character of the (001) and (110) surfaces of NiO. Most notably, for the (110) NiO surface we observe a remarkable electronic state characterized by an alternating charge-transfer and Mott-Hubbard character of the band gap in the surface and subsurface NiO layers, respectively. This novel form of electronic order stabilized by strong correlations is not driven by lattice reconstructions but of purely electronic origin. We notice the importance of orbital differentiation of the Ni $e_{g}$ states to characterize the Mott-Hubbard insulating state of the (001) and (110) NiO surfaces. The unoccupied Ni $e_{g}$ surface states are seen to split from the lower edge of the conduction band to form strongly localized states in the fundamental gap of bulk NiO. Our results for the surface energies of the (001) and (110) NiO surfaces show that the (001) facet of $\mathrm{NiO}$ has significantly lower energy. This implies that the relative stability of different surfaces, at least from a purely energetic point of view, does not depend on the presence or absence of magnetic order in $\mathrm{NiO}$.
\end{abstract}

DOI: 10.1103/PhysRevB.103.165108

\section{INTRODUCTION}

The series of transition metal monoxides $\mathrm{MnO}, \mathrm{FeO}, \mathrm{CoO}$, and $\mathrm{NiO}$ with an electronic configuration ranging from $3 d^{5}$ to $3 d^{8}$, respectively, has attracted much attention due to their diverse electronic and magnetic properties [1-5], allowing for a broad range of applications, e.g., in electronics and spintronics [6-9], energy storage [10-13], and heterogeneous catalysis [14-17]. At low temperature, these compounds exhibit a correlated Mott-Hubbard or charge-transfer insulating behavior with a large band gap of $\sim 2-4 \mathrm{eV}$, associated with a strong localization of the $3 d$ electrons [18-22]. Below the Néel temperature, ranging from $T_{N} \sim 116$ to $523 \mathrm{~K}$ for $\mathrm{MnO}$

\footnotetext{
*Corresponding author: ivan.v.leonov@yandex.ru

${ }^{\dagger}$ Corresponding author: silke.biermann@teorfys.lu.se
}

Published by the American Physical Society under the terms of the Creative Commons Attribution 4.0 International license. Further distribution of this work must maintain attribution to the author(s) and the published article's title, journal citation, and DOI. Funded by Bibsam. to $\mathrm{NiO}$, respectively, these materials display an antiferromagnetic type-II long-range magnetic ordering and undergo a structural phase transition from a rocksalt cubic to a distorted rhombohedral ( $\mathrm{MnO}$ and $\mathrm{NiO})$ or monoclinic $(\mathrm{FeO}$ and $\mathrm{CoO})$ phase [23].

Over the past decades particular attention has been devoted to understanding the nature of the band gap and excitation spectrum of $\mathrm{MnO}-\mathrm{NiO}$ [18-22,24-30]. In fact, due to the strongly correlated nature of electron interactions between the $3 d$ electrons, theoretical computations of the electronic structure of these materials using band-structure methods are particularly difficult. The Coulomb interactions may be modelled by including an onsite Hubbard parameter $U$ to treat the effect of correlations in the partially filled $3 d$ shell, e.g., within the so-called DFT $+U$ method [25-27]. While these "beyond standard density functional theory (DFT)" methods often give a reliable description of the electronic properties such as band gaps, magnetic moments, and lattice displacements [30-35], these methods neglect electron dynamics and hence cannot capture correlated electron phenomena related to a Mott transition such as a coherence-incoherence crossover, quasiparticle behavior, and strong quasiparticle mass renormalization [18,19,36-38]. In fact, to determine 
the electronic properties of these materials one needs to go beyond conventional band-structure methods to include dynamical correlation effects of the $3 d$ electron (e.g., using DFT $+U$ subject to dynamical symmetry-breaking spin and structural effects $[28,29])$.

It has been shown that the dynamical correlations and strong localization of the $d$ (and $f$ ) electrons can be described by employing a DFT + dynamical mean-field theory $($ DFT + DMFT) approach [36-38]. DFT + DMFT makes it possible to treat local correlation effects in a self-consistent, numerically exact way, providing a good description of the electronic and magnetic properties of correlated models and materials [36-63]. By using DFT + DMFT it becomes possible to compute the material-specific properties of complex correlated materials, e.g., to determine the electronic structure, magnetic state, and crystal structure of (para-) magnetic materials at finite temperatures, e.g., near the Mott transition [44-46,51-55].

The DFT + DMFT approach has been widely used to study the physical properties of the bulk structure of transition metal monoxides (TMOs), providing a quantitative description of the electronic, magnetic, and structural properties of these materials [43-53,55-60]. While the electronic structure and magnetic state of bulk TMOs are relatively well established nowadays (see, however, Refs. [64-66]), this is not the case for the different surface structures of metal oxides and their interfaces [1-5]. In fact, the electronic structure of a surface or interface can differ significantly from that of bulk, since their electronic states are responsible for a variety of novel (emerging) physical properties. In the case of surfaces, this affects, e.g., molecular adsorption and reactions that determine the catalytic processes at the surface [1-3]. In this respect, $\mathrm{NiO}$ is of particular interest among other TMOs due to its importance as catalysts for electrochemical applications, fuel cells, and batteries [10-17] (in addition to being a model system for understanding the Mott-Hubbard metal-insulator transition [18,19]).

The electronic properties of the nonpolar (001) and (110) and polar (111) $\mathrm{NiO}$ surfaces have been actively studied using different experimental techniques, such as x-ray photoemission spectroscopy (XPS), x-ray linear magnetic dichroism, electron-energy-loss spectroscopy (EELS), and scanning tunneling microscopy (STM), that give detailed information about the surface $d$-shell excitations and atomically resolved images of the surface states and reconstructions [1-5,67-74]. On the other hand, the effects of electron correlations and the electronic structure and magnetic properties of the $\mathrm{NiO}$ surfaces are still poorly understood. In practice, the electronic and magnetic properties of $\mathrm{NiO}$ surfaces have been studied using band-structure methods with the static mean-field Hubbard $U$ treatment of correlation effects in the $\mathrm{Ni} 3 d$ shell (e.g., within DFT $+U)$ [75-84]. The strong localization of the $3 d$ electrons and the finite temperature (para-) magnetic behavior of $\mathrm{NiO}$ still pose a challenge for an accurate description of the electronic properties of its surfaces using, e.g, DFT + DMFT $[85,86]$, which is crucial for potential technical applications [10-17].

In this paper, we employ a multisite extension $[41,42,54,87,88]$ of the DFT + DMFT method [89] implemented within a plane-wave pseudopotential formalism
[51-54] to explore the effects of electronic correlations on the electronic properties of the nonpolar paramagnetic (PM) (001) and (110) surfaces of NiO. We study the electronic structure, magnetic properties, and surface energies of the (001) and (110) $\mathrm{NiO}$ with particular attention given to the effect of structural confinement and its influence on the strength of electronic correlations in the PM (001) and (110) $\mathrm{NiO}$.

\section{COMPUTATIONAL DETAILS}

We perform a DFT + DMFT study of the electronic structure, magnetic properties, and surface energies of the clean (001) and (110) surfaces of the prototypical correlated insulator NiO. In DFT we employ the generalized gradient Perdew-Burke-Ernzerhof approximation (GGA) [90] within plane-wave pseudopotential formalism [91]. Our DFT + DMFT calculations explicitly include the $\mathrm{Ni} 3 d$ and $\mathrm{O} 2 p$ valence states by constructing a basis set of atomic-centered Wannier functions within the energy window spanned by the $p$ - $d$ band complex [92]. This makes it possible to take into account a charge transfer between the $\mathrm{Ni} 3 d$ and $\mathrm{O}$ $2 p$ states, accompanied by the strong onsite Coulomb correlations in the $\mathrm{Ni} 3 d$ shell. In order to solve the realistic many-body problem within DMFT we use the continuoustime hybridization-expansion quantum Monte-Carlo (QMC) algorithm [93].

The DFT + DMFT calculations are performed in the paramagnetic state at an electronic temperature $T=390 \mathrm{~K}$. We use the average Hubbard $U=10 \mathrm{eV}$ and Hund's exchange $J=1 \mathrm{eV}$, in accordance with previous works [25,52,57,94]. The Coulomb interaction $U$ and Hund's $J$ have been treated in the density-density approximation, with no spin-orbit coupling contribution. We employ the fully localized doublecounting correction evaluated from the self-consistently determined local occupations to account for the electronic interactions already described by DFT. We use a fully selfconsistent in charge density implementation of DFT + DMFT in order to take into account the effects of charge redistribution caused by electronic correlations and electron-lattice coupling $[52,95]$. In order to study the effects of structural confinement and relaxations we employ a multi-impurity-site extension of the DFT + DMFT method in order to treat correlations in the $3 d$ bands of the structurally distinct Ni sites [41,42,54,87,88]. In addition, we perform structural relaxations of the surface and subsurface states of $\mathrm{NiO}$ within DFT. The DFT structural relaxation calculations are performed for the (fictitious) ferromagnetic (FM) state of $\mathrm{NiO}$. For the relaxed structures the electronic properties of (001) and (110) PM NiO were evaluated within DFT + DMFT. The spectral functions were computed using the maximum entropy method and the Padé analytical continuation procedure.

\section{RESULTS AND DISCUSSION}

\section{A. Electronic structure of (001) $\mathrm{NiO}$}

We begin with the electronic structure and equilibrium lattice volume calculations of bulk $\mathrm{NiO}$ in the paramagnetic state using DFT + DMFT [51,52,89]. In agreement with experiment, our calculations yield a correlated insulator with 
(a) $(001) \mathrm{NiO}$
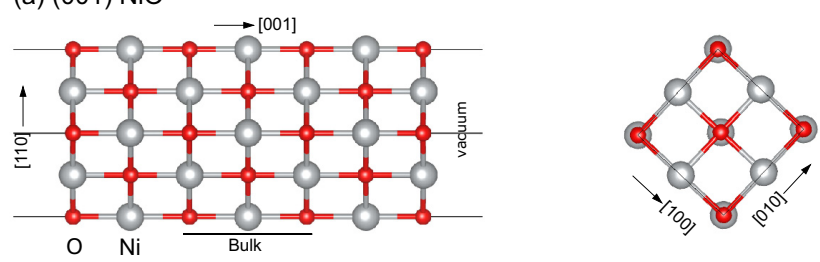

(b) $(110) \mathrm{NiO}$
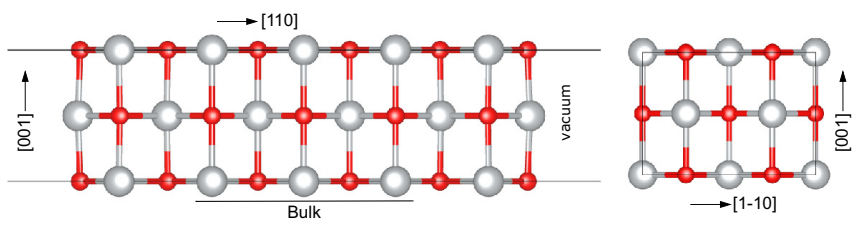

FIG. 1. (001) $\mathrm{NiO}$ (a) and (110) $\mathrm{NiO}$ slabs (b) used in the present DFT + DMFT calculations [96].

a large $\mathrm{Ni} d$ - $d$ energy gap of about $2.9 \mathrm{eV}$ and equilibrium lattice constant $a=4.233 \AA$ (lattice volume of $\sim 128$ a.u. ${ }^{3}$ and bulk modulus $187 \mathrm{GPa}$ ). The $\mathrm{Ni}^{2+} d^{8}$ ions are in a high-spin $(S=1)$ state with an instantaneous local magnetic moment $\sqrt{\left\langle\hat{m}_{z}^{2}\right\rangle} \simeq 1.83 \mu_{\mathrm{B}}$, corresponding to the fluctuating moment of $1.7 \mu_{\mathrm{B}}$. The latter is evaluated as the imaginary-time average of the local spin susceptibilities $\chi(\tau)=\left\langle\hat{m}_{z}(\tau) \hat{m}_{z}(0)\right\rangle$ as $M_{\text {loc }}=\left(k_{B} T \int \chi(\tau) d \tau\right)^{1 / 2}$. This result is in good agreement with the experimental estimate of 1.7-1.9 $\mu_{\mathrm{B}}$ [97]. We note that the top of the valence band of $\mathrm{NiO}$ has a mixed $\mathrm{Ni} e_{g}$ and $\mathrm{O} 2 p$ character, with a resonant peak in the filled $\mathrm{Ni}$ $e_{g}$ band located at about $1 \mathrm{eV}$ below the Fermi level. The latter can be ascribed to the formation of a Zhang-Rice bound state [98]. Our results suggest a mixture of a Mott-Hubbard $\mathrm{Ni} d-d$ and charge transfer type of the band gap which is caused by the effect of Coulomb correlations in the $\mathrm{Ni} 3 d$ shell $[20,21,24]$. The bottom of the conduction band is mainly of the $\mathrm{Ni} 3 d$ character with a broad paraboliclike $\mathrm{Ni} 4 s$ band at the Brillouin zone $\Gamma$ point.

Next, we use the equilibrium lattice constant obtained by DFT + DMFT for bulk NiO to construct the (001) symmetric slab consisting of 7-NiO monolayers (ML), with a thickness of about $12.59 \AA$ and vacuum of $\sim 21 \AA$ [see Fig. 1(a)]. Each ML in the slab contains two Ni sites. The top two NiO MLs are considered as a surface and subsurface, respectively, with 3-ML-thick $\mathrm{NiO}$ as a quasibulk. In our calculations we set the kinetic energy cutoff for the plane wave basis to 65 Ryd for the wave function and 650 Ryd for the charge density. We employ the three-impurity-site DFT + DMFT to treat structurally distinct the surface, subsurface, and quasibulk $\mathrm{Ni} 3 d$ ions [99]. We first perform structural optimization of the surface and subsurface (001) $\mathrm{NiO}$ layers within FM GGA. It gives a small inward relaxation of the top ML of $\delta_{12} \simeq-2.5 \%(0.054 \AA)$ and a weak outward relaxation of the subsurface ML $\delta_{23} \sim 0.6 \%$, with nearly absent surface buckling. Here, surface relaxations are characterized as a percent change of the spacing between layers $i$ and $j$ versus the equilibrium interlayer spacing $d_{0}, \delta_{i j}=\left(z_{i}^{\mathrm{Ni}}-z_{j}^{\mathrm{Ni}}\right) / d_{0}$. We also find a small inward interplane relaxation between the first and third MLs, $\delta_{13} \sim-1.0 \%$. Interestingly, the nomagnetic GGA yields $-2.7 \%$ and $-2.4 \%$ relaxation for the top and sub-

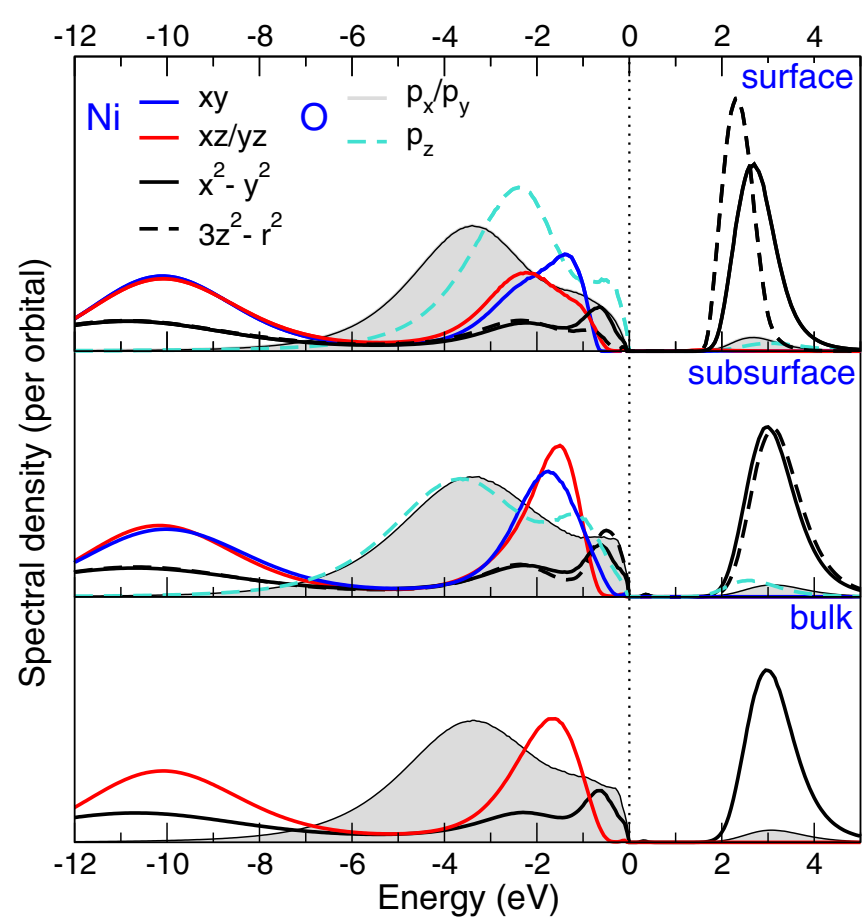

FIG. 2. Ni $3 d$ and O $2 p$ spectral functions calculated by DFT + DMFT for the relaxed (001) $\mathrm{NiO}$ at a temperature $T=390 \mathrm{~K}$.

surface NiO-MLs, respectively, with a small surface buckling of $\sim 0.11 \AA$. At the same time, a structural optimization of the antiferromagnetic (001) surface of $\mathrm{NiO}$ within spin-polarized DFT $+U$ (with $U-J=7.0 \mathrm{eV}$ ) $[26,27,91]$ gives a relatively weak inward interplane relaxation of the surface layer $\delta_{12} \sim$ $-1.05 \%(\sim 0.022 \AA)$, accompanied by a small outward relaxation of the subsurface ML $\delta_{23} \sim 0.5 \%$ and inward relaxation between the first and third MLs, $\delta_{13} \sim-0.3 \%$.

In Fig. 2 we show the orbitally-resolved spectral functions of (001) NiO obtained by DFT + DMFT for the relaxed within FM GGA surface of $\mathrm{NiO}$. Our results for the unrelaxed bulk-terminated (001) $\mathrm{NiO}$ are shown in Supplemental Material Fig. S1 [100]. In accordance with experiment, we find a Mott-Hubbard insulator with a large (fundamental) energy gap of about $2.1 \mathrm{eV}$. The calculated local magnetic moments are $1.84,1.82$, and $1.82 \mu_{B}$ for the surface, subsurface, and quasibulk Ni ions, respectively. The corresponding fluctuating moments are $1.78,1.74$, and $1.75 \mu_{\mathrm{B}}$. Our results reveal a weak charge redistribution between the surface and bulk $\mathrm{Ni}$ sites. The total Wannier Ni $3 d$ occupancies are nearly the same for all the $\mathrm{Ni}$ sites and differ by less than 0.03 . In addition, we find no sizable distinction between the $\mathrm{Ni} 3 z^{2}-r^{2}$ and $x^{2}-y^{2}$ orbital occupancies, which differ by less than 0.03 for all the $\mathrm{Ni}$ sites. At the same time, we observe a substantial splitting of the occupied $\mathrm{O} 2 p$ and the empty Ni $e_{g}$ bands at the surface caused by the effect of surface symmetry breaking (see the top panel of Fig. 2). It leads to a sizable reduction of the band gap from about $2.6 \mathrm{eV}$ in the quasibulk to $2.1 \mathrm{eV}$ at the surface. Moreover, surface relaxation (strain) is seen to result in a small increase of the band gap by less than $\sim 5 \%$, from 2.0 to $2.1 \mathrm{eV}$. This result underlines the importance of the crystal-field splitting caused by the effect of surface truncation 


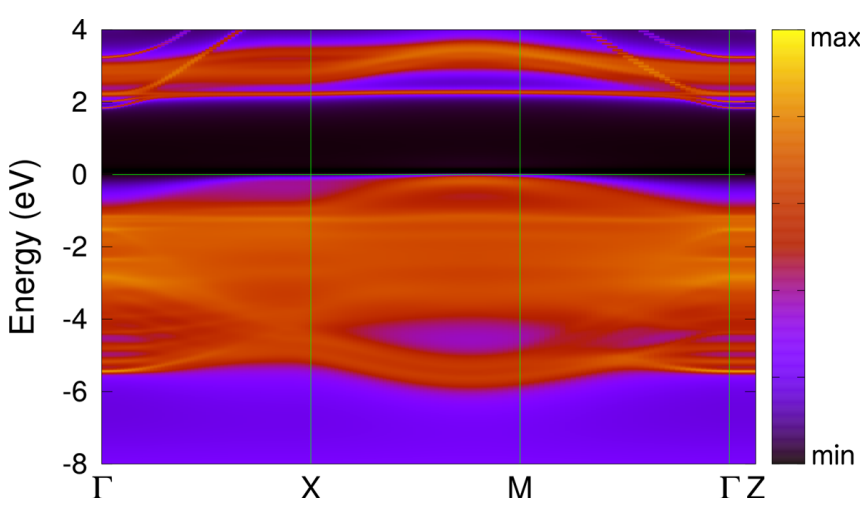

FIG. 3. The k-resolved total spectral function $A(\mathbf{k}, \omega)$ of (001) $\mathrm{NiO}$ along the $\Gamma-\mathrm{X}-\mathrm{M}-\Gamma-\mathrm{Z}$ lines in the Brillouin zone as obtained by DFT + DMFT at a temperature $T=390 \mathrm{~K}$.

and relaxations for establishing the Mott insulating state of (001) NiO.

In the surface layer the top of the $(001) \mathrm{NiO}$ valence band shows a mixed $\mathrm{Ni} x^{2}-y^{2}$ and $\mathrm{O} 2 p_{z}$ character, with a large contribution from the $\mathrm{O} 2 p$ states, caused by the surface symmetry breaking and strain effects. Moreover, in the subsurface ML the top of the valence band has a mixed Ni $3 z^{2}-r^{2}$ and $\mathrm{O} 2 p_{x} / p_{y}$ character. Our results therefore suggest a charge-transfer character of the (001) surface. Moreover, we also notice the importance of orbital differentiation of the Ni $e_{g}$ states (for the surface and subsurface NiO-MLs) to characterize the correlated insulating state of (001) NiO. In addition to this, the unoccupied $\mathrm{Ni} 3 z^{2}-r^{2}$ surface states are seen to split from the lower edge of the conduction band and form a strongly localized (and dispersionless) band at about $2 \mathrm{eV}$ above the $E_{F}$ [see our results for the k-resolved spectra of (001) $\mathrm{NiO}$ in Fig. 3]. In contrast to this, at the top of the valence band, all bands merge with the continuum of bulk states.

We also calculated the local (dynamical) susceptibility $\chi(\tau)=\left\langle\hat{m}_{z}(\tau) \hat{m}_{z}(0)\right\rangle$ for the surface, subsurface, and quasibulk $\mathrm{Ni}^{2+}$ ions of PM (001) NiO. In Fig. 4(a) we display our results for the $\mathrm{Ni} e_{g}$ contributions in $\chi(\tau) . \tau$ denotes
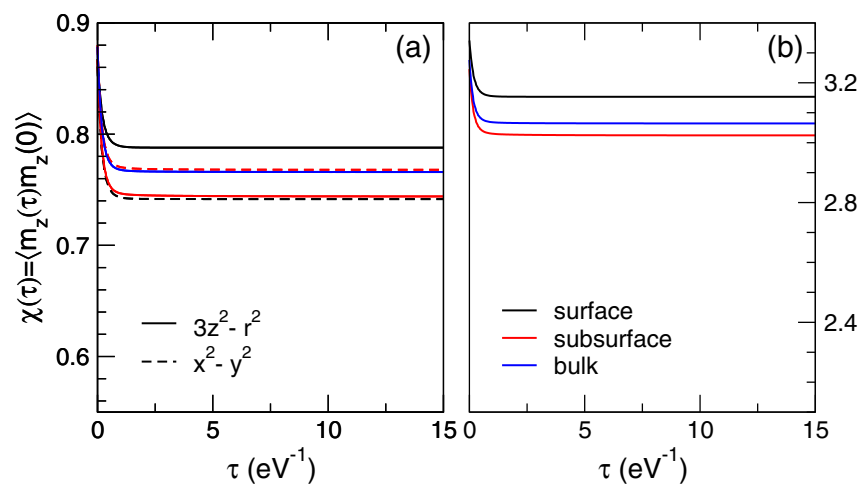

FIG. 4. Orbital-dependent (a) and total (b) local spin susceptibility $\chi(\tau)=\left\langle\hat{m}_{z}(\tau) \hat{m}_{z}(0)\right\rangle$ for the surface, subsurface, and bulk of paramagnetic $(001) \mathrm{NiO}$ as obtained by DFT + DMFT at an inverse temperature $\beta=1 / k_{B} T=30 \mathrm{eV}^{-1}$. Note that $\chi(\tau)$ is symmetric with respect to $\beta / 2, \chi(\tau) \equiv \chi(\beta-\tau)$. imaginary times. The value of this quantity around $\tau=\beta / 2$ indicates the long time limit, and deviations from this value for small and large $\beta$ indicate strong magnetic fluctuations. In the present case, all the $e_{g}$ contributions are seen to be almost independent of $\tau$, suggesting that the $3 d$ electrons are localized to form fluctuating local moments. In fact, $\chi(\tau)$ is seen to be nearly constant and close to its maximal value $S=1$ for the Ni $e_{g}$ states. However, in the surface layer $\chi(\tau)$ is seen to be remarkably larger at all $\tau$, implying higher localization of the surface $e_{g}$ states. This leads to a higher charge-transfer character of the (001) $\mathrm{NiO}$ surface, while for the bulk our results suggest a mixture of a Mott-Hubbard $d$ - $d$ and charge transfer character of the band gap [20,21,24].

For the (001) $\mathrm{NiO}$ we compute the surface energy within DFT + DMFT as $\gamma \simeq\left(E_{\text {slab }}^{N}-N E_{\text {bulk }}\right) /(2 S)$ [101]. Here, $E_{\text {slab }}^{N}$ is the total energy of an $N$-formula-unit slab of $\mathrm{NiO}$ $E_{\text {bulk }}$ is the total energy of bulk $\mathrm{NiO}$ per formula unit (f.u.). $S$ is the surface area and the factor two accounts for the two surfaces in the slab unit cell. In order to minimize numerical differences between the bulk and the slab DFT + DMFT total energy calculations we adopt the same setup parameters within DFT + DMFT (such as kinetic energy cutoffs, k-point sampling, the shape of the unit cell, etc.) [102]. In particular, in our DFT + DMFT calculations we used a $10 \times 10 \times 8$ k-point grid for the (001) slab containing 14 f.u. of NiO. To estimate $E_{\text {bulk }}$, we computed the 4-f.u. cubic supercell of bulk $\mathrm{NiO}$ with a $10 \times 10 \times 28 \mathbf{k}$ grid. In both calculations we employed the same kinetic energy cutoffs for the wave functions (65 Ry) and for the charge density and potentials (650 Ry). Our result $59 \mathrm{meV} / \AA^{2}$ is in good agreement with previous estimates in the antiferromagnetically ordered phase $[78,80,83]$. We note that the nonmagnetic GGA yields $44 \mathrm{meV} / \AA^{2}$.

\section{B. (110) NiO surface}

We now turn to our results for the PM (110) NiO. To model (110) $\mathrm{NiO}$ we design the (110) 11-ML-thick slab with symmetry-equivalent surfaces. It is of $14.59 \AA$ thick, with a vacuum spacer of about $21 \AA$ [see Fig. 1(b)]. In our DFT + DMFT calculations, we treat the top three NiO MLs as surface, subsurface, and sub-subsurface layers, respectively. The remaining 5-ML-thick layer in the middle is taken as quasibulk NiO. We use the four-impurity-site extension of DFT + DMFT to treat the effect of correlations in the $3 d$ shell of the structurally distinct Ni ions. In DFT the kinetic energy cutoff for the plane wave basis was set to 50 Ryd for the wave function and 500 Ryd for the charge density. All the calculations are performed in the local basis set determined by diagonalization of the corresponding $\mathrm{Ni} 3 d$ occupation matrices. Structural optimization of the surface and two subsurface (110) NiO layers within FM GGA suggests that surface relaxations in (110) $\mathrm{NiO}$ are long range. It gives a large inward relaxation of the top layer of about $-11.0 \%(-0.16 \AA)$ accompanied by a sizable outward relaxation of the subsurface layer by $3.4 \%$. The interplane relaxation between the first and third layer $\delta_{13}$ is about $-3.8 \%$, while $\delta_{15} \sim-2.9 \%$. The above results are consistent with the results of a structural optimization of antiferromagnetic (110) $\mathrm{NiO}$ within $\mathrm{DFT}+U$ [91]. In particular, it gives a large inward interplane relaxation of the surface layer $\delta_{12} \sim-9.2 \%(-0.138 \AA)$, accompanied by a 


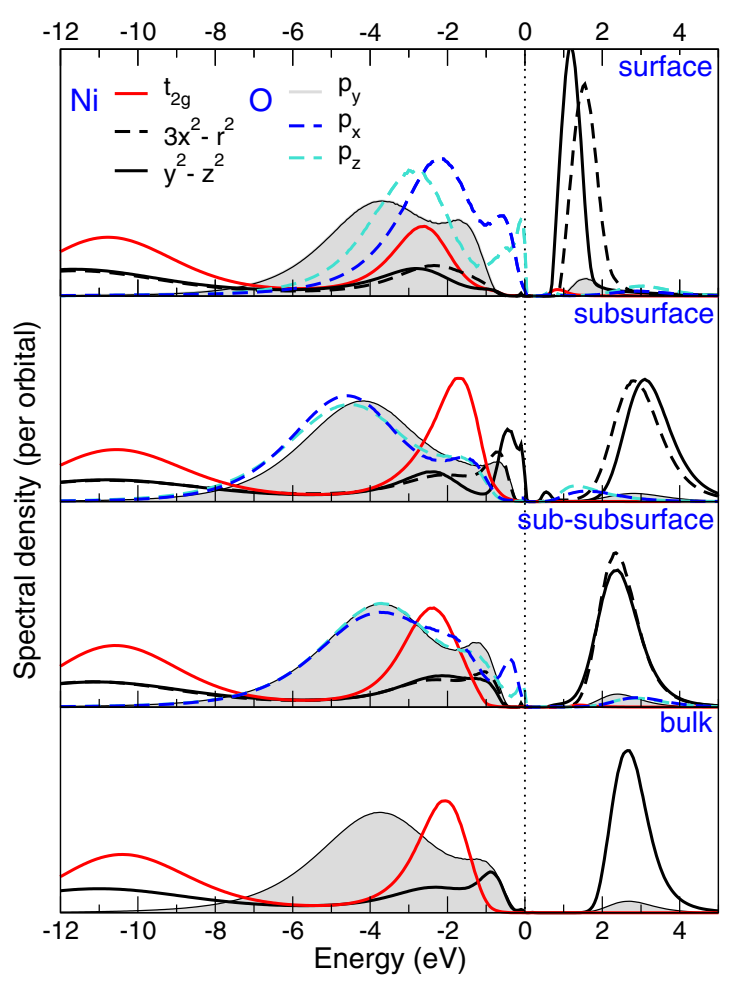

FIG. 5. Ni $e_{g}$ and O $2 p$ spectral functions of paramagnetic (110) $\mathrm{NiO}$ obtained by DFT + DMFT at a temperature $T=390 \mathrm{~K}$.

large outward relaxation of the subsurface layer $\delta_{23} \sim 5.46 \%$ $(0.082 \AA)$ and relatively weak inward relaxations between the first and third MLs, $\delta_{13} \sim-1.88 \%$, and the third and fourth MLs, $\delta_{34} \sim-0.59 \%$.

In Fig. 5 we summarize our results for the $\mathrm{Ni} 3 d$ and $\mathrm{O}$ $2 p$ spectral functions of PM (110) NiO. Our results for the k-resolved spectra are shown in Fig. 6. We obtain a MottHubbard insulating solution with a relatively large band gap of $0.9 \mathrm{eV}$. Similarly to (001) $\mathrm{NiO}$, we observe a weak variation of the local moments among the different Ni layers. In particular, the calculated local magnetic moments are $1.83,1.79,1.82$, and $1.82 \mu_{\mathrm{B}}$ for the surface, two subsurfaces, and quasibulk $\mathrm{Ni}$ ions, respectively. Our estimate of the fluctuating moments is $1.77,1.70,1.73$, and $1.75 \mu_{\mathrm{B}}$. The $\mathrm{Ni} 3 d$ occupancies are

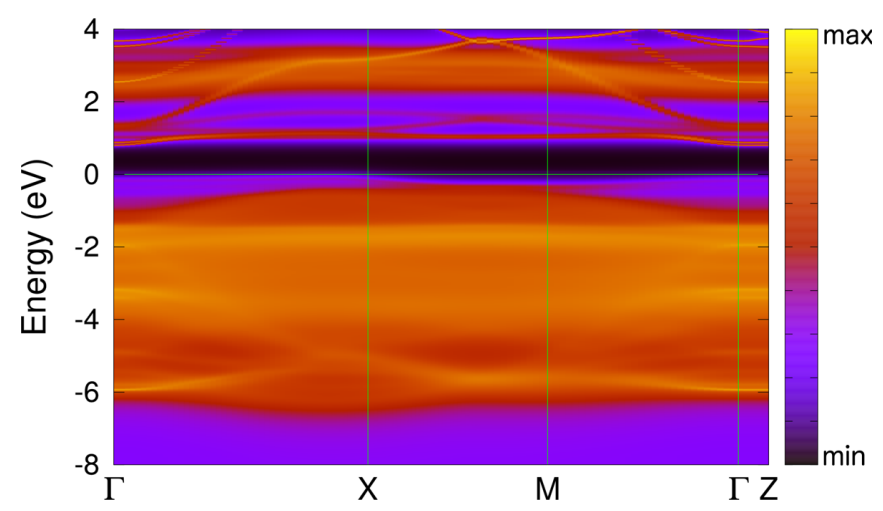

FIG. 6. The k-resolved total spectral function $A(\mathbf{k}, \omega)$ of (110) $\mathrm{NiO}$ along the $\Gamma-\mathrm{X}-\mathrm{M}-\Gamma-\mathrm{Z}$ lines in the Brillouin zone as obtained by DFT + DMFT at a temperature $T=390 \mathrm{~K}$. nearly the same for all the Ni sites, differing by less than 0.04. In addition, we observe no sizable difference between the Ni $3 x^{2}-r^{2}$ and $y^{2}-z^{2}$ orbital occupancies in (110) $\mathrm{NiO}$. The deviations are less than 0.03 . Our analysis of the layer-dependent $\mathrm{Ni} 3 d$ and $\mathrm{O} 2 p$ spectral functions reveals a remarkable reduction of the band gap at the surface layer. In fact, it drops by about three times, from $2.9 \mathrm{eV}$ in the quasibulk to $0.9 \mathrm{eV}$ in the surface layer, in the relaxed (110) NiO. Most importantly, our result for the (fundamental) gap value of (110) $\mathrm{NiO}$ is more than twice smaller than that in the (001) $\mathrm{NiO}(2.1 \mathrm{eV})$. In close similarity to $(001) \mathrm{NiO}$, structural relaxations of the surface result in an increase of the band gap from 0.8 to $0.9 \mathrm{eV}$.

Our results for the Ni $3 d$ and O $2 p$ spectral functions show a sizable splitting of the occupied $\mathrm{O} 2 p$ and the empty $\mathrm{Ni}$ $e_{g}$ bands (see Figs. 5 and 6). While at the top of the valence band, all bands merge with the continuum of bulk states, the unoccupied $\mathrm{Ni} e_{g}$ surface states are seen to split from the lower edge of the conduction band. These $\mathrm{Ni} e_{g}$ states form a localized band with a bandwidth of about $0.4 \mathrm{eV}$ which appears in the bulk band gap at about $1 \mathrm{eV}$ (see Fig. 6). Moreover, a detailed analysis of the top of the valence band reveals a large contribution of the $\mathrm{O} 2 p$ states near the $E_{F}$, which are strongly mixed with the $\mathrm{Ni} 3 d$ states. It is interesting to note, however, that the situation significantly differs from that in the (001) $\mathrm{NiO}$ or in bulk NiO. In fact, in contrast to the bulk and (001) $\mathrm{NiO}$ we observe that the top of the valence bands of (110) $\mathrm{NiO}$ has almost pure $\mathrm{O} 2 p$ character at the surface and in the sub-subsurface layer, implying a pure charge-transfer type of the band gap of the (110) surface of NiO. In contrast to this, at the subsurface layer, it is nearly purely of $\mathrm{Ni} 3 d$ $\left(y^{2}-z^{2}\right)$ character, suggestive of a Mott-Hubbard type of the band gap in the subsurface layer of (110) $\mathrm{NiO}$. Our results therefore document a novel electronic state characterized by an alternating pure charge-transfer and Mott-Hubbard character of the band gap in the surface and subsurface (110) $\mathrm{NiO}$, respectively. This behavior suggests the absence of prominent Zhang-Rice physics at the (110) surface of $\mathrm{NiO}$ that reappear in bulk $\mathrm{NiO}[43,47,52]$. We notice that this remarkable alternating charge-transfer and Mott-Hubbard state is robust with respect to the lattice reconstructions of the (110) surface as seen in the spectral function of the bulk-terminated (110) $\mathrm{NiO}$ (see Supplemental Material Fig. S2). This suggests that it is of purely electronic origin. Moreover, we notice a remarkable orbital differentiation of the Ni $e_{g}$ states at the (sub)surface which seems to be important to characterize the transport properties of (110) $\mathrm{NiO}$.

In Fig. 7 we display our results for the layer-dependent orbitally-resolved and total local susceptibility $\chi(\tau)$. We observe that all the $e_{g}$ contributions are seen to be almost independent of $\tau$, suggesting strongly localized nature of the $\mathrm{Ni} e_{g}$ states in (110) NiO. Similarly to (001) $\mathrm{NiO}$ the surface layer $\chi(\tau)$ is somewhat larger for all $\tau$, implying a higher degree of localization of the surface $e_{g}$ states. On the other hand, counterintuitive to this is the reduction of the band gap at the surface layer seen in the Ni $3 d$ and $\mathrm{O} 2 p$ spectral function for the (110) NiO surface, which is caused by charge-transfer effects.

We also calculate the surface energy of the relaxed (110) $\mathrm{NiO}$ using DFT + DMFT. In DFT + DMFT we used a $10 \times$ 


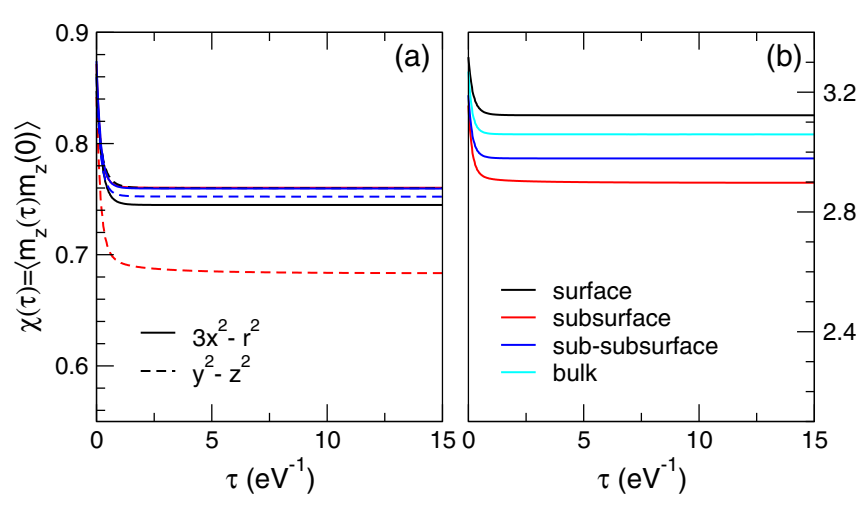

FIG. 7. Orbital-dependent (a) and total (b) local spin susceptibility $\chi(\tau)=\left\langle\hat{m}_{z}(\tau) \hat{m}_{z}(0)\right\rangle$ of paramagnetic (110) $\mathrm{NiO}$ as obtained by DFT + DMFT.

$10 \times 4$ k-point grid for the 22-f.u. slab calculation of (110) $\mathrm{NiO}$. In the bulk calculation we employed a 8-f.u. tetragonal supercell with a $10 \times 10 \times 24 \mathbf{k}$ grid. For the slab and bulk DFT + DMFT calculations the kinetic energy cutoffs were taken 50 Ry for the wave functions and 500 Ry for the charge density and potentials. Our result for the surface energy of (110) $\mathrm{NiO} 125 \mathrm{meV} / \AA^{2}$ is substantially larger than that for the (001) $\mathrm{NiO}\left(59 \mathrm{meV} / \AA^{2}\right)$, implying that (001) NiO has significantly lower energy facet. The nonmagnetic GGA calculation gives $85 \mathrm{meV} / \AA^{2}$ for (110) $\mathrm{NiO}$.

\section{CONCLUSIONS}

In conclusion, we have calculated the electronic structure, magnetic properties, and surfaces energies of the (001) and (110) surfaces of the prototypical correlated insulating material, PM NiO, using a multisite extension of the DFT + DMFT method. Our results reveal a complex interplay between electronic correlations and surface effects in $\mathrm{NiO}$. We obtain that the electronic structure of the (001) and (110) $\mathrm{NiO}$ differs significantly from that of bulk NiO. In both (001) and (110) $\mathrm{NiO}$ we observe a sizable reduction of the band gap at the surface. The latter is most significant for the (110) $\mathrm{NiO}$, from 2.9 in the quasibulk to $0.9 \mathrm{eV}$ at the surface. Surface relaxations (surface strain) are seen to result in a remarkable increase of the band gap in comparison with the bulk-terminated $\mathrm{NiO}$.

We observe a substantial splitting of the $\mathrm{O} 2 p$ and $\mathrm{Ni} e_{g}$ bands at the surface caused by the effect of surface symmetry breaking. Our results suggest a charge-transfer character of the (001) and (110) surfaces of NiO. Most importantly, for the (110) $\mathrm{NiO}$ surface we observe a remarkable electronic state characterized by an alternating pure charge-transfer and Mott-Hubbard character of the band gap in the surface and subsurface $\mathrm{NiO}$ layers, respectively. This novel form of electronic order stabilized by strong correlations is not driven by lattice reconstructions but of purely electronic origin. Moreover, we notice the importance of orbital-differentiation of the $\mathrm{Ni} e_{g}$ states to characterize the Mott insulating state of the
(001) and (110) NiO. The unoccupied $\mathrm{Ni} e_{g}$ surface states are seen to split from the lower edge of the conduction band to form strongly localized states in the fundamental gap of bulk $\mathrm{NiO}$.

Our DFT + DMFT calculations reveal a remarkable difference between the electronic structure of the (001) and (110) $\mathrm{NiO}$ surfaces. For example, we obtain a large difference in their (fundamental) band gap value (by about a factor of two) due to the surface effects, $\sim 2.1 \mathrm{eV}$ in the (001) and $0.9 \mathrm{eV}$ for the (110) $\mathrm{NiO}$ surfaces. This suggests a higher catalytic activity of the (110) $\mathrm{NiO}$ surface than that of the (001) $\mathrm{NiO}$ surface. In agreement with previous estimates in the antiferromagnetically ordered phase, our DFT + DMFT calculations yield significantly different surface energies for the (001) and (110) $\mathrm{NiO}$ surfaces, of 59 and $125 \mathrm{meV} / \AA^{2}$, respectively. Thus, the (001) $\mathrm{NiO}$ surface is found to have a significantly lower energy facet. We find that the effect of electron-electron correlations in $\mathrm{NiO}$ results in a sizable enhancement of the $\mathrm{NiO}$ surface energies by $34-48 \%$ with respect to the nonmagnetic GGA. Overall, our results for the electronic structure, magnetic properties, and surface energies of (001) and (110) $\mathrm{NiO}$ agree well with experimental data measured in the antiferromagnetic phase. Our results further reveal an intriguing independence of properties such as the energetical hierarchy of different surfaces of the presence or absence of antiferromagnetic order in $\mathrm{NiO}$.

In accordance with previous studies of surfaces of strongly correlated materials, e.g., applications of DFT + DMFT to study $\mathrm{SrVO}_{3}$ and $\mathrm{CaVO}_{3}$ thin films $[62,63]$ and to a $\mathrm{SrTiO}_{3}$ surface [61], our results demonstrate the ability of multisite DFT + DMFT to compute the electronic structure and magnetic properties of transition metal oxide surfaces and thin films. The DFT + DMFT computations can be used to promote our understanding of the physics of surfaces, which are promising for applications. Further studies could, for example, deal with $\mathrm{NiO}$ deposited on $\mathrm{SrTiO}_{3}$, elucidating the increased reactivity with water of such systems [103] or the design of highly active catalysts, e.g., for developing hybrid nickel-zinc and zinc-air batteries [13]. $\mathrm{NiO}$ is also being actively investigated for its resistive switching properties [104-106] for the description of which extensions of DFT + DMFT might also prove useful.

\section{ACKNOWLEDGMENTS}

We thank S. Backes, S. Panda, D. D. Sarma, and I. A. Abrikosov for valuable discussions. The DFT/DFT $+U$ calculations and theoretical analysis of the electronic properties of (001) and (110) $\mathrm{NiO}$ were supported by the state assignment of Minobrnauki of Russia (theme "Electron" No. AAAAA18-118020190098-5). The DFT + DMFT calculations of magnetic properties of (001) and (110) $\mathrm{NiO}$ were supported by Russian Science Foundation (Project No. 19-72-30043). S.B. acknowledges support from the European Research Council under Grant Agreement No. 617196, Project CORRELMAT, and from IDRIS/GENCI Orsay under Project No. t2021091393.
[1] H. H. Kung, Transition Metal Oxides: Surface Chemistry and Catalysis (Elsevier, Amsterdam, 1989), Vol. 45.
[2] V. E. Henrich and P. A. Cox, The Surface Science of Metal Oxides (Cambridge University Press, Cambridge, 1994). 
[3] C. Noguera, Physics and Chemistry of Oxide Surfaces (Cambridge University Press, Cambridge, 1996).

[4] H.-J. Freund, H. Kuhlenbeck, and V. Staemmler, Oxide surfaces, Rep. Prog. Phys. 59, 283 (1996).

[5] U. Kaiser, A. Schwarz, and R. Wiesendanger, Magnetic exchange force microscopy with atomic resolution, Nature (London) 446, 522 (2007).

[6] Q. Liu, Q. Chen, Q. Zhang, Y. Xiao, X. Zhong, G. Dong, M.-P. Delplancke-Ogletree, H. Terryn, K. Baert, F. Reniers, and X. Diao, In situ electrochromic efficiency of a nickel oxide thin film: origin of electrochemical process and electrochromic degradation, J. Mater. Chem. C 6, 646 (2018).

[7] W. Lin, K. Chen, S. Zhang, and C. L. Chien, Enhancement of Thermally Injected Spin Current through an Antiferromagnetic Insulator, Phys. Rev. Lett. 116, 186601 (2016).

[8] E. Aytan, B. Debnath, F. Kargar, Y. Barlas, M. M. Lacerda, J. X. Li, R. K. Lake, J. Shi, and A. A. Balandin, Spin-phonon coupling in antiferromagnetic nickel oxide, Appl. Phys. Lett. 111, 252402 (2017).

[9] M. Dabrowski, T. Nakano, D. M. Burn, A. Frisk, D. G. Newman, C. Klewe, Q. Li, M. Yang, P. Shafer, E. Arenholz, T. Hesjedal, G. van der Laan, Z. Q. Qiu, and R. J. Hicken, Coherent Transfer of Spin Angular Momentum by Evanescent Spin Waves within Antiferromagnetic NiO, Phys. Rev. Lett. 124, 217201 (2020).

[10] P. L. S. G. Poizot, S. Laruelle, S. Grugeon, L. Dupont, and J. M. Tarascon, Nano-sized transition-metal oxides as negative-electrode materials for lithium-ion batteries, Nature (London) 407, 496 (2000).

[11] M. V. Reddy, G. V. Subba Rao, and B. V. R. Chowdari, Metal oxides and oxysalts as anode materials for $\mathrm{Li}$ ion batteries, Chem. Rev. 113, 5364 (2013).

[12] D. Su, M. Ford, and G. Wang, Mesoporous NiO crystals with dominantly exposed (110) reactive facets for ultrafast lithium storage, Sci. Rep. 2, 924 (2012).

[13] D. U. Lee, J. Fu, M. G. Park, H. Liu, A. G. Kashkooli, and Z. Chen, Self-assembled $\mathrm{NiO} / \mathrm{Ni}(\mathrm{OH})_{2}$ nanoflakes as active material for high-power and high-energy hybrid rechargeable battery, Nano Lett. 16, 1794 (2016).

[14] W. Zhao, M. Bajdich, S. Carey, and A. Vojvodic, Water dissociative adsorption on $\mathrm{NiO}(111)$ : Energetics and structure of the hydroxylated surface, ACS Catal. 6, 7377 (2016).

[15] A. Nelson, K. E. Fritz, S. Honrao, R. G. Hennig, R. D. Robinson, and J. Suntivich, Increased activity in hydrogen evolution electrocatalysis for partial anionic substitution in cobalt oxysulfide nanoparticles, J. Mater. Chem. A 4, 2842 (2016).

[16] R. Poulain, A. Klein, and J. Proost, Electrocatalytic properties of (001)-, (110)-, and (111)-oriented NiO thin films toward the oxygen evolution reaction, J. Phys. Chem. C 122, 22252 (2018).

[17] S. Gong, A. Wang, Y. Wang, H. Liu, N. Han, and Y. Chen, Heterostructured $\mathrm{Ni} / \mathrm{NiO}$ nanocatalysts for ozone decomposition, ACS Appl. Nano Mater. 3, 597 (2020).

[18] N. F. Mott, Insulator Transitions (Taylor \& Francis, London, 1990).

[19] M. Imada, A. Fujimori, and Y. Tokura, Metal-insulator transitions, Rev. Mod. Phys. 70, 1039 (1998).

[20] G. A. Sawatzky and J. W. Allen, Magnitude and Origin of the Band Gap in NiO, Phys. Rev. Lett. 53, 2339 (1984).
[21] J. Zaanen, G. A. Sawatzky, and J. W. Allen, Band Gaps and Electronic Structure of Transition-Metal Compounds, Phys. Rev. Lett. 55, 418 (1985).

[22] Z.-X. Shen, R. S. List, D. S. Dessau, B. O. Wells, O. Jepsen, A. J. Arko, R. Barttlet, C. K. Shih, F. Parmigiani, J. C. Huang, and P. A. P. Lindberg, Electronic structure of NiO: Correlation and band effects, Phys. Rev. B 44, 3604 (1991).

[23] W. L. Roth, Magnetic structures of $\mathrm{MnO}, \mathrm{FeO}, \mathrm{CoO}$, and $\mathrm{NiO}$, Phys. Rev. 110, 1333 (1958).

[24] T. M. Schuler, D. L. Ederer, S. Itza-Ortiz, G. T. Woods, T. A. Callcott, and J. C. Woicik, Character of the insulating state in NiO: A mixture of charge-transfer and Mott-Hubbard character, Phys. Rev. B 71, 115113 (2005).

[25] V. I. Anisimov, J. Zaanen, and O. K. Andersen, Band theory and Mott insulators: Hubbard $U$ instead of Stoner $I$, Phys. Rev. B 44, 943 (1991); V. I. Anisimov, I. V. Solovyev, M. A. Korotin, M. T. Czyzyk, and G. A. Sawatzky, Densityfunctional theory and $\mathrm{NiO}$ photoemission spectra, ibid. 48, 16929 (1993).

[26] S. L. Dudarev, G. A. Botton, S. Y. Savrasov, C. J. Humphreys and A. P. Sutton, Electron-energy-loss spectra and the structural stability of nickel oxide: An LSDA $+U$ study, Phys. Rev. B 57, 1505 (1998).

[27] M. Cococcioni and S. de Gironcoli, Linear response approach to the calculation of the effective interaction parameters in the LDA $+U$ method, Phys. Rev. B 71, 035105 (2005).

[28] G. Trimarchi, Z. Wang, and A. Zunger, Polymorphous band structure model of gapping in the antiferromagnetic and paramagnetic phases of the Mott insulators $\mathrm{MnO}, \mathrm{FeO}, \mathrm{CoO}$, and NiO, Phys. Rev. B 97, 035107 (2018); J. Varignon, M. Bibes, and A. Zunger, Origin of band gaps in $3 d$ perovskite oxides, Nat. Commun. 10, 1658 (2019).

[29] Z. Wang, O. Malyi, X. Zhao, and A. Zunger, Mass enhancement in $3 d$ and $s-p$ perovskites from symmetry breaking, arXiv:2006.00199.

[30] V. Potapkin, L. Dubrovinsky, I. Sergueev, M. Ekholm, I. Kantor, D. Bessas et al., Magnetic interactions in $\mathrm{NiO}$ at ultrahigh pressure, Phys. Rev. B 93, 201110(R) (2016).

[31] A. Schrön, C. Rödl, and F. Bechstedt, Crystalline and magnetic anisotropy of the $3 d$-transition metal monoxides $\mathrm{MnO}, \mathrm{FeO}$, CoO, and NiO, Phys. Rev. B 86, 115134 (2012).

[32] C. Rödl and F. Bechstedt, Optical and energy-loss spectra of the antiferromagnetic transition metal oxides $\mathrm{MnO}, \mathrm{FeO}, \mathrm{CoO}$, and $\mathrm{NiO}$ including quasiparticle and excitonic effects, Phys. Rev. B 86, 235122 (2012).

[33] H. Jiang, R. I. Gomez-Abal, P. Rinke, and M. Scheffler, First-principles modeling of localized $d$ states with the GW@LDA + $U$ approach, Phys. Rev. B 82, 045108 (2010).

[34] K. Karlsson, F. Aryasetiawan, and O. Jepsen, Method for calculating the electronic structure of correlated materials from a truly first-principles LDA $+U$ scheme, Phys. Rev. B 81, 245113 (2010).

[35] P. Liu, C. Franchini, M. Marsman, and G. Kresse, Assessing model-dielectric-dependent hybrid functionals on the antiferromagnetic transition-metal monoxides $\mathrm{MnO}, \mathrm{FeO}, \mathrm{CoO}$, and NiO, J. Phys.: Condens. Matter 32, 015502 (2020).

[36] A. Georges, G. Kotliar, W. Krauth, and M. J. Rozenberg, Dynamical mean-field theory of strongly correlated fermion systems and the limit of infinite dimensions, Rev. Mod. Phys. 68, 13 (1996); G. Kotliar, S. Y. Savrasov, K. Haule, V. S. 
Oudovenko, O. Parcollet, and C. A. Marianetti, Electronic structure calculations with dynamical mean-field theory, ibid. 78, 865 (2006).

[37] R. C. Kent and G. Kotliar, Toward a predictive theory of correlated materials, Science 361, 348 (2018).

[38] S. Biermann, Topics in Current Chemistry (Springer, Berlin, Heidelberg, 2014), Vol. 347, pp. 303-345.

[39] S. Biermann, A. Dallmeyer, C. Carbone, W. Eberhardt, C. Pampuch, O. Rader, M. I. Katsnelson, A. I. Lichtenstein, Observation of Hubbard bands in $\gamma$-manganese, JETP Lett. 80, 612 (2004).

[40] E. Pavarini, S. Biermann, A. Poteryaev, A. I. Lichtenstein, A. Georges, and O. K. Andersen, Mott Transition and Suppression of Orbital Fluctuations in Orthorhombic $3 d^{1}$ Perovskites, Phys. Rev. Lett. 92, 176403 (2004).

[41] A. I. Poteryaev, A. I. Lichtenstein, and G. Kotliar, Nonlocal Coulomb Interactions and Metal-Insulator Transition in $\mathrm{Ti}_{2} \mathrm{O}_{3}$ : A Cluster LDA+DMFT Approach, Phys. Rev. Lett. 93, 086401 (2004).

[42] S. Biermann, A. I. Poteryaev, A. I. Lichtenstein, and A. Georges, Dynamical Singlets and Correlation-Assisted Peierls Transition in $\mathrm{VO}_{2}$, Phys. Rev. Lett. 94, 026404 (2005).

[43] J. Kunes, V. I. Anisimov, A. V. Lukoyanov, and D. Vollhardt, Local correlations and hole doping in NiO: A dynamical mean-field study, Phys. Rev. B 75, 165115 (2007); J. Kunes, V. I. Anisimov, S. L. Skornyakov, A. V. Lukoyanov, and D. Vollhardt, NiO: Correlated Band Structure of a ChargeTransfer Insulator, Phys. Rev. Lett. 99, 156404 (2007).

[44] J. Kunes, A. V. Lukoyanov, V. I. Anisimov, R. T. Scalettar, and W. E. Pickett, Collapse of magnetic moment drives the Mott transition in MnO, Nat. Mater. 7, 198 (2008).

[45] A. O. Shorikov, Z. V. Pchelkina, V. I. Anisimov, S. L. Skornyakov, and M. A. Korotin, Orbital-selective pressuredriven metal to insulator transition in $\mathrm{FeO}$ from dynamical mean-field theory, Phys. Rev. B 82, 195101 (2010).

[46] K. Ohta, R. E. Cohen, K. Hirose, K. Haule, K. Shimizu, and Y. Ohishi, Experimental and Theoretical Evidence for PressureInduced Metallization in $\mathrm{FeO}$ with Rocksalt-Type Structure, Phys. Rev. Lett. 108, 026403 (2012).

[47] Q. Yin, A. Gordienko, X. Wan, S. Y. Savrasov, Calculated Momentum Dependence of Zhang-Rice States in Transition Metal Oxides, Phys. Rev. Lett. 100, 066406 (2008).

[48] P. Thunström, I. Di Marco, and O. Eriksson, Electronic Entanglement in Late Transition Metal Oxides, Phys. Rev. Lett. 109, 186401 (2012).

[49] K. Byczuk, J. Kunes, W. Hofstetter, and D. Vollhardt, Quantification of Correlations in Quantum Many-Particle Systems, Phys. Rev. Lett. 108, 087004 (2012).

[50] I. A. Nekrasov, N. S. Pavlov, M. V. Sadovskii, Consistent LDA'+DMFT - an unambiguous way to avoid double counting problem: NiO test, JETP Lett. 95, 581 (2012); Consistent LDA'+DMFT approach to electronic structure of transition metal oxides: charge transfer insulators and correlated metals, J. Exp. Theor. Phys. 116, 620 (2013).

[51] I. Leonov, Metal-insulator transition and local-moment collapse in $\mathrm{FeO}$ under pressure, Phys. Rev. B 92, 085142 (2015); I. Leonov, A. V. Ponomareva, R. Nazarov, and I. A. Abrikosov, Pressure-induced spin-state transition of iron in magnesiowüstite (Fe, Mg)O, ibid. 96, 075136 (2017).
[52] I. Leonov, L. Pourovskii, A. Georges, and I. A. Abrikosov, Magnetic collapse and the behavior of transition metal oxides at high pressure, Phys. Rev. B 94, 155135 (2016); I. Leonov, A. O. Shorikov, V. I. Anisimov, and I. A. Abrikosov, Emergence of quantum critical charge and spin-state fluctuations near the pressure-induced Mott transition in $\mathrm{MnO}, \mathrm{FeO}, \mathrm{CoO}$, and $\mathrm{NiO}$, ibid. 101, 245144 (2020).

[53] E. Greenberg, R. Nazarov, A. Landa, J. Ying, R. Q. Hood, B. Hen, R. Jeanloz, V. B. Prakapenka, V. V. Struzhkin, G Kh. Rozenberg, and I. Leonov, Phase transitions and spinstate of iron in $\mathrm{FeO}$ at the conditions of Earth's deep interior, arXiv:2004.00652.

[54] E. Greenberg, I. Leonov, S. Layek, Z. Konopkova, M. P. Pasternak, L. Dubrovinsky, R. Jeanloz, I. A. Abrikosov, and G. Kh. Rozenberg, Pressure-Induced Site-Selective Mott Insulator-Metal Transition in $\mathrm{Fe}_{2} \mathrm{O}_{3}$, Phys. Rev. X 8, 031059 (2018); I. Leonov, G. Kh. Rozenberg, and I. A. Abrikosov, Charge disproportionation and site-selective local magnetic moments in the post-perovskite-type $\mathrm{Fe}_{2} \mathrm{O}_{3}$ under ultra-high pressures, npj Comput. Mater. 5, 90 (2019).

[55] N. Lanatá, T.- H. Lee, Y.-X. Yao, V. Stevanović, and V. Dobrosavljević, Connection between Mott physics and crystal structure in a series of transition metal binary compounds, npj Comput. Mater. 5, 30 (2019).

[56] S. K. Panda, B. Pal, S. Mandal, M. Gorgoi, S. Das, I. Sarkar et al., High photon energy spectroscopy of NiO: Experiment and theory, Phys. Rev. B 93, 235138 (2016).

[57] S. K. Panda, H. Jiang, and S. Biermann, Pressure dependence of dynamically screened Coulomb interactions in NiO: Effective Hubbard, Hund, intershell, and intersite components, Phys. Rev. B 96, 045137 (2017).

[58] F. Lechermann, W. Körner, D. F. Urban, and C. Elsässer, Interplay of charge-transfer and Mott-Hubbard physics approached by an efficient combination of self-interaction correction and dynamical mean-field theory, Phys. Rev. B 100, 115125 (2019).

[59] S. Mandal, K. Haule, K. M. Rabe, and D. Vanderbilt, Influence of magnetic ordering on the spectral properties of binary transition metal oxides, Phys. Rev. B 100, 245109 (2019).

[60] E. Koemets, I. Leonov, M. Bykov, E. Bykova, S. Chariton et al., Revealing the Complex Nature of Bonding in the Binary High-Pressure Compound $\mathrm{FeO}_{2}$, Phys. Rev. Lett. 126, 106001 (2021).

[61] Z. Zhong, A. Tóth, and K. Held, Theory of spin-orbit coupling at $\mathrm{LaAlO}_{3} / \mathrm{SrTiO}_{3}$ interfaces and $\mathrm{SrTiO}_{3}$ surfaces, Phys. Rev. B 87, 161102(R) (2013).

[62] Z. Zhong, M. Wallerberger, J. M. Tomczak, C. Taranto, N. Parragh, A. Toschi, G. Sangiovanni, and K. Held, Electronics with Correlated Oxides: $\mathrm{SrVO}_{3} / \mathrm{SrTiO}_{3}$ as a Mott Transistor, Phys. Rev. Lett. 114, 246401 (2015).

[63] S. Beck, G. Sclauzero, U. Chopra, and C. Ederer, Metalinsulator transition in $\mathrm{CaVO}_{3}$ thin films: Interplay between epitaxial strain, dimensional confinement, and surface effects, Phys. Rev. B 97, 075107 (2018).

[64] M. Karolak, G. Ulm, T. Wehling, V. Mazurenko, A. Poteryaev, and A. Lichtenstein, Double counting in LDA+DMFT - the example of NiO, J. Electron. Spectrosc. Relat. Phenom. 181, 11 (2010). 
[65] T. Haupricht, J. Weinen, A. Tanaka, R. Gierth, S. G. Altendorf, Y.-Y. Chin et al., Local correlations, non-local screening, multiplets, and band formation in $\mathrm{NiO}$, arXiv:1210.6675.

[66] C.-Y. Kuo, T. Haupricht, J. Weinen, H. Wu, K.-D. Tsuei, M. W. Haverkort, A. Tanaka, and L. H. Tjeng, Challenges from experiment: Electronic structure of NiO, Eur. Phys. J.: Spec. Top. 226, 2445 (2017).

[67] B. Fromme, M. Möller, Th. Anschütz, C. Bethke, and E. Kisker, Electron-Exchange Processes in the Excitations of NiO(001) Surface $d$ States, Phys. Rev. Lett. 77, 1548 (1996).

[68] D. Alders, L. H. Tjeng, F. C. Voogt, T. Hibma, G. A. Sawatzky, C. T. Chen, J. Vogel, M. Sacchi, and S. Iacobucci, Temperature and thickness dependence of magnetic moments in $\mathrm{NiO}$ epitaxial films, Phys. Rev. B 57, 11623 (1998).

[69] J. Stöhr, A. Scholl, T. J. Regan, S. Anders, J. Lüning, M. R. Scheinfein, H. A. Padmore, and R. L. White, Images of the Antiferromagnetic Structure of a NiO(001) Surface by Means of X-Ray Magnetic Linear Dichroism Spectromicroscopy, Phys. Rev. Lett. 83, 1862 (1999).

[70] A. Barbier, C. Mocuta, H. Kuhlenbeck, K. F. Peters, B. Richter, and G. Renaud, Atomic Structure of the Polar NiO(111)- $p(2 \times 2)$ Surface, Phys. Rev. Lett. 84, 2897 (2000); A. Barbier, C. Mocuta, and G. Renaud, Structure, transformation, and reduction of the polar NiO (111) surface, Phys. Rev. B 62, 16056 (2000).

[71] H. Ohldag, A. Scholl, F. Nolting, S. Anders, F. U. Hillebrecht, and J. Stöhr, Spin Reorientation at the Antiferromagnetic $\mathrm{NiO}(001)$ Surface in Response to an Adjacent Ferromagnet, Phys. Rev. Lett. 86, 2878 (2001).

[72] F. U. Hillebrecht, H. Ohldag, N. B. Weber, C. Bethke, U. Mick, M. Weiss, and J. Bahrdt, Magnetic Moments at the Surface of Antiferromagnetic NiO(001), Phys. Rev. Lett. 86, 3419 (2001).

[73] L. Soriano, I. Preda, A. Gutiérrez, S. Palacin, M. Abbate, and A. Vollmer, Surface effects in the Ni $2 p$ x-ray photoemission spectra of NiO, Phys. Rev. B 75, 233417 (2007).

[74] F. Pielmeier and F. J. Giessibl, Spin Resolution and Evidence for Superexchange on $\mathrm{NiO}(001)$ Observed by Force Microscopy, Phys. Rev. Lett. 110, 266101 (2013).

[75] M. R. Castell, S. L. Dudarev, G. A. D. Briggs, and A. P. Sutton, Unexpected differences in the surface electronic structure of $\mathrm{NiO}$ and $\mathrm{CoO}$ observed by STM and explained by first-principles theory, Phys. Rev. B 59, 7342 (1999).

[76] J.-T. Hoeft, M. Kittel, M. Polcik, S. Bao, R. L. Toomes, J.-H. Kang, D. P. Woodruff, M. Pascal, and C. L. A. Lamont, Molecular Adsorption Bond Lengths at Metal Oxide Surfaces: Failure of Current Theoretical Methods, Phys. Rev. Lett. 87, 086101 (2001).

[77] D. Ködderitzsch, W. Hergert, W. M. Temmerman, Z. Szotek, A. Ernst, and $\mathrm{H}$. Winter, Exchange interactions in $\mathrm{NiO}$ and at the NiO(001) surface, Phys. Rev. B 66, 064434 (2002).

[78] O. Bengone, M. Alouani, J. Hugel, P. Blöchl, LDA + U calculated electronic and structural properties of $\mathrm{NiO}(001)$ and $\mathrm{NiO}(111) p(2 \times 2)$ surfaces, Comput. Mater. Sci. 24, 192 (2002).

[79] A. Wander, I. J. Bush, and N. M. Harrison, Stability of rocksalt polar surfaces: An ab initio study of $\mathrm{MgO}$ (111) and $\mathrm{NiO}$ (111), Phys. Rev. B 68, 233405 (2003).

[80] A. Rohrbach, J. Hafner, and G. Kresse, Molecular adsorption on the surface of strongly correlated transition-metal oxides: A case study for CO/NiO(100), Phys. Rev. B 69, 075413 (2004); A. Rohrbach and J. Hafner, Molecular adsorption of NO on $\mathrm{NiO}(001)$ : DFT and DFT $+U$ calculations, ibid. 71, 045405 (2005).

[81] A. M. Ferrari, C. Pisani, F. Cinquini, L. Giordano, and G. Pacchioni, Cationic and anionic vacancies on the $\mathrm{NiO}(001)$ surface: DFT $+U$ and hybrid functional density functional theory calculations, J. Chem. Phys. 127, 174711 (2007).

[82] N. Yu, W.-B. Zhang, N. Wang, Y.-F. Wang, and B.-Y. Tang, Water Adsorption on a $\mathrm{NiO}(001)$ Surface: A GGA + U Study, J. Phys. Chem. C 112, 452 (2008).

[83] A. Schrön, M. Granovskij, and F. Bechstedt, Influence of onsite Coulomb interaction $U$ on properties of $\mathrm{MnO}(001) 2 \times 1$ and $\mathrm{NiO}(001) 2 \times 1$ surfaces, J. Phys.: Condens. Matter 25, 094006 (2013); A. Schrön and F. Bechstedt, Spin-dependent properties and images of $\mathrm{MnO}, \mathrm{FeO}, \mathrm{CoO}$, and $\mathrm{NiO}(001)$ surfaces, Phys. Rev. B 92, 165112 (2015).

[84] V. S. C. Kolluru and R. G. Hennig, Role of magnetism on transition metal oxide surfaces in vacuum and solvent, Phys. Rev. Materials 4, 045803 (2020).

[85] M. Potthoff and W. Nolting, Surface metal-insulator transition in the Hubbard model, Phys. Rev. B 59, 2549 (1999); Metallic surface of a Mott insulator-Mott insulating surface of a metal, 60, 7834 (1999).

[86] M. Potthoff, Metal-Insulator Transitions at Surfaces, Adv. Solid State Phys. 42, 121 (2002).

[87] J.-Z. Ma, A. van Roekeghem, P. Richard, Z.-H. Liu, H. Miao, L.-K. Zeng et al., Correlation-Induced Self-Doping in the IronPnictide Superconductor $\mathrm{Ba}_{2} \mathrm{Ti}_{2} \mathrm{Fe}_{2} \mathrm{As}_{4} \mathrm{O}$, Phys. Rev. Lett. 113, 266407 (2014).

[88] P. Delange, T. Ayral, S. I. Simak, M. Ferrero, O. Parcollet, S. Biermann, and L. Pourovskii, Large effects of subtle electronic correlations on the energetics of vacancies in $\alpha$-Fe, Phys. Rev. B 94, 100102(R) (2016).

[89] The DFT+DMFT code employed in this study is available upon request from the corresponding author I.L. (ivan.v.leonov@yandex.ru).

[90] J. P. Perdew, K. Burke, and M. Ernzerhof, Generalized Gradient Approximation Made Simple, Phys. Rev. Lett. 77, 3865 (1996).

[91] S. Baroni, S. de Gironcoli, A. Dal Corso, and P. Giannozzi, Rev. Mod. Phys. 73, 515 (2001); P. Giannozzi, S. Baroni, N. Bonini, M. Calandra, R. Car et al., QUANTUM ESPRESSO: A modular and open-source software project for quantum simulations of materials, J. Phys.: Condens. Matter 21, 395502 (2009).

[92] N. Marzari, A. A. Mostofi, J. R. Yates, I. Souza, and D. Vanderbilt, Maximally localized Wannier functions: Theory and applications, Rev. Mod. Phys. 84, 1419 (2012); V. I. Anisimov, D. E. Kondakov, A. V. Kozhevnikov, I. A. Nekrasov, Z. V. Pchelkina, J. W. Allen et al., Full orbital calculation scheme for materials with strongly correlated electrons, Phys. Rev. B 71, 125119 (2005); G. Trimarchi, I. Leonov, N. Binggeli, Dm. Korotin, and V. I. Anisimov, LDA+DMFT implemented with the pseudopotential plane-wave approach, J. Phys.: Condens. Matter 20, 135227 (2008).

[93] E. Gull, A. J. Millis, A. I. Lichtenstein, A. N. Rubtsov, M. Troyer, and P. Werner, Continuous-time Monte Carlo methods for quantum impurity models, Rev. Mod. Phys. 83, 349 (2011). 
[94] R. Sakuma and F. Aryasetiawan, First-principles calculations of dynamical screened interactions for the transition metal oxides $M \mathrm{O}(M=\mathrm{Mn}, \mathrm{Fe}, \mathrm{Co}, \mathrm{Ni})$, Phys. Rev. B 87, 165118 (2013).

[95] For a review, see, e.g., L. V. Pourovskii, B. Amadon, S. Biermann, and A. Georges, Self-consistency over the charge density in dynamical mean-field theory: A linear muffin-tin implementation and some physical implications, Phys. Rev. B 76, 235101 (2007); K. Haule, Quantum Monte Carlo impurity solver for cluster dynamical mean-field theory and electronic structure calculations with adjustable cluster base, ibid. 75, 155113 (2007); B. Amadon, F. Lechermann, A. Georges, F. Jollet, T. O. Wehling, and A. I. Lichtenstein, Plane-wave based electronic structure calculations for correlated materials using dynamical mean-field theory and projected local orbitals, ibid. 77, 205112 (2008); M. Aichhorn, L. Pourovskii, V. Vildosola, M. Ferrero, O. Parcollet, T. Miyake, A. Georges, and S. Biermann, Dynamical mean-field theory within an augmented plane-wave framework: Assessing electronic correlations in the iron pnictide $\mathrm{LaFeAsO}$, ibid. 80, 085101 (2009); B. Amadon, A self-consistent DFT+DMFT scheme in the projector augmented wave method: Applications to cerium, $\mathrm{Ce}_{2} \mathrm{O}_{3}$ and $\mathrm{Pu}_{2} \mathrm{O}_{3}$ with the Hubbard I solver and comparison to DFT $+U$, J. Phys.: Condens. Matter 24, 075604 (2012).

[96] K. Momma and F. Izumi, VESTA 3 for three-dimensional visualization of crystal, volumetric and morphology data, J. Appl. Crystallogr. 44, 1272 (2011).

[97] H. A. Alperin, J. Phys. Soc. Jpn. Suppl. B 17, 12 (1962); B. E. F. Fender, A. J. Jacobson, and F. A. Wedgwood, Covalency Parameters in $\mathrm{MnO}, \alpha-\mathrm{MnS}$, and $\mathrm{NiO}$, J. Chem. Phys. 48, 990 (1968); A. K. Cheetham and D. A. O. Hope, Magnetic ordering and exchange effects in the antiferromagnetic solid solutions $\mathrm{Mn}_{x} \mathrm{Ni}_{1-x} \mathrm{O}$, Phys. Rev. B 27, 6964 (1983).

[98] F. C. Zhang and T. M. Rice, Effective hamiltonian for the superconducting Cu oxides, Phys. Rev. B 37, 3759 (1988).
[99] S. Keshavarz, Y. O. Kvashnin, I. Di Marco, A. Delin, M. I. Katsnelson, A. I. Lichtenstein, and O. Eriksson, Layerresolved magnetic exchange interactions of surfaces of late $3 d$ elements: Effects of electronic correlations, Phys. Rev. B 92, 165129 (2015).

[100] See Supplemental Material at http://link.aps.org/ supplemental/10.1103/PhysRevB.103.165108 for additional results supporting our conclusions.

[101] N. E. Singh-Miller and N. Marzari, Surface energies, work functions, and surface relaxations of low-index metallic surfaces from first principles, Phys. Rev. B 80, 235407 (2009).

[102] We note that even on the DFT or DFT $+U$ level, it is difficult to estimate the surface energies reliably. In fact, it requires estimating a relatively small total energy difference between the slab and bulk total energies, which depend sensitively upon fine details of the total energy calculations (kinetic energy cutoffs, k-points sampling, etc.). In our DFT+DMFT calculations (for given cutoffs in kinetic energy, charge density, Matsubara frequencies, and k-points sampling) the total energy is typically converged to $\sim 1-2 \mathrm{meV} /$ f.u., which makes the stochastic errors in QMC to be essentially negligible. At the same time, we had to converge the DFT+DMFT results with different k-points sets.

[103] S. Gerhold, M. Riva, Z. Wang, R. Bliem, M. Wagner, J. Osiecki, K. Schulte, M. Schmid, and U. Diebold, NickelOxide-Modified $\mathrm{SrTiO}_{3}(110)-(4 \times 1)$ Surfaces and Their Interaction with Water, J. Phys. Chem. C 119, 20481 (2015).

[104] A. Sawa, Resistive Switching in transition metal oxides, Mater. Today 11, 28 (2008).

[105] K. Kinoshtia, T. Okutani, H. Tanaka, T. Hinoki, K. Yazawa, K. Ohmi, and S. Kishida, Opposite bias polarity dependence of resistive switching in $n$-type Ga-doped- $\mathrm{ZnO}$ and $p$-type $\mathrm{NiO}$ thin films, Appl. Phys. Lett. 96, 143505 (2010).

[106] K. M. Kim, D. S. Jeong, and C. S. Hwang, Nanofilamentary resistive switching in binary oxide system; a review on the present status and outlook, Nanotech. 22, 254002 (2011). 